\section{CPS-292 EFFECTIVENESS AND SAFETY OF IBRUTINIB IN CHRONIC LYMPHATIC LEUKAEMIA: MULTICENTRE STUDY}

'B Serna Serrano, ${ }^{2}$ Casas Hidalgo, 'M Díaz Rangel, ${ }^{1}$ A Valladolid Walsh*, ${ }^{1} F$ Sánchez Rubio, ${ }^{1} \mathrm{M}$ Clemente Andújar, ${ }^{1} \mathrm{~S}$ Plata Paniagua, ${ }^{1} \mathrm{~S}$ Ruiz Sánchez. ${ }^{1}$ Complejo Hospitalario Universitario De Albacete, Pharmacy, Albacete, Spain; ${ }^{2}$ Hospital De Hellin, Pharmacy, Hellin, Spain

10.1136/ejhpharm-2021-eahpconf.124

Background and importance Ibrutinib is a potent Burton tyrosine kinase inhibitor involved in the proliferation and survival of chronic lymphatic leukaemia (CLL) B cells. This study was mainly motivated by suspensions for toxicity.

Aim and objectives The objective was to analyse the effectiveness and safety of ibrutinib in CLL.

Material and methods This was a retrospective observational study including all patients with CLL treated with ibrutinib until September 2020 from two hospitals. Data were obtained from Farmatools and clinical records. SPSS Statistics V.17.0 was used for statistical analysis. The analysed variables were demographic and clinical data $(\mathrm{D} / \mathrm{C})$, treatment, effectiveness and safety.

- $\mathrm{D} / \mathrm{C}$ : number of patients; sex; age; the presence of mutations in chromosome TP53 (mut-TP53) and deletion of chromosome 17 (del17p); progression to Richter (PR).

- Treatment: previous lines (PL $\mathrm{PLRUTINIB}_{\mathrm{IB}}$ ); duration of treatment (DT IBRUTINIB).

- Effectiveness: progression free-survival (PFS) and overall survival (OS) using Kaplan-Meyer statistical analysis.

- Safety: the most frequent number, type and degree of adverse events (AE) according to the common terminology criteria for adverse events (CTCAE) V.4.03 and necessary treatment modifications (TM) (dose reduction (DR), treatment suspension (TS), both (DR-TS)).

Results

- D/C: 60 patients (58\% men); median age 80 years (55-98); mut-TP53 (8.3\%), del17p (15\%); PR (8.3\%).

- Treatment: $\mathrm{PL}_{\text {IBRUTINIB }}(56 \%$ one-third, $3 \% \geq$ four); median DT $_{\text {IBRUTINIB }} 13.5$ months (1-53).

- Effectiveness: at 12-24 months, PFS was 79.3-72.8\%; OS was $85.1-76.4 \%$. Mean values obtained were $x P F S=38$ months \pm 3.3 (95\% CI 31.7 to 44.5 ) and $x O S=40.5$ months \pm 2.9 (95\% CI 34.8 to 46.2 ).

- Safety: the most frequent $\mathrm{AE}(\geq 15 \%)$ were diarrhoea, pneumonia, skin rash and haematomas. The most frequent G3-4 AE ( $\geq 5 \%)$ were neutropenia, pneumonia, skin rash, anaemia and atrial fibrillation. A 53\% TM by AE: 23\% TS, 19\% DR-TS and 11\% DR.

Conclusion and relevance The effectiveness and safety results obtained were similar to those of pivotal studies (PS). PFS and $O S$ at 12-24 months in our study $(79.3-72.8 \%$ and $85.1-76.4 \%)$ were lower than the results of PS (89.8$82.3 \%$ and $90.2-89.6 \%)$. With regard to the safety data, PS showed lower dropout rates due to AE (6\% vs 23\%) and lower dose reductions ( $8 \%$ vs 19\%) although the toxicity profile and the most frequent G3-4 AEs were similar to the PS.

\section{REFERENCES AND/OR ACKNOWLEDGEMENTS}

1. doi: $10.1056 /$ NEJMoa1400376

2. doi: 10.1056/NEJMoa1215637
3. https://www.ema.europa.eu/en/documents/assessment-report/imbruvica-epar-public-assessment-report en.pdf

Conflict of interest No conflict of interest

\section{CPS-293 EFFICACY AND SAFETY OF CYCLIN DEPENDENT KINASE INHIBITORS IN METASTATIC BREAST CANCER}

L Cantarelli*, JA Morales Barrios, S Garcia Gil, B Del Rosario Garcia, GJ Nazco Casariego, F Gutierrez Nicolas. Complejo Hospitalario Universitario De Canarias, Pharmacy, Santa Cruz De Tenerife, Spain

\subsection{6/ejhpharm-2021-eahpconf.125}

Background and importance Cyclin dependent kinase inhibitors (CDKs) have demonstrated efficacy in the treatment of hormone receptor positive $(\mathrm{RH}+)$ and human epidermal growth factor receptor 2 (HER2-) metastatic breast cancer.

Aim and objectives To evaluate the efficacy and safety of cyclin inhibitor drugs (CDK) in patients with metastatic breast cancer.

Material and methods An 18 month, single centre, retrospective study (January 2019 to June 2020) that included all patients with metastatic breast cancer treated with palbociclib $(\mathrm{PB})$, ribociclib $(\mathrm{RB})$ and abemaciclib $(\mathrm{AB})$ was conducted. The following variables were collected: age, sex, time of disease follow-up, time of treatment with the drug, neoadjuvant/adjuvant and previous hormonal therapy. Efficacy was evaluated by calculating progression free survival (PFS) for each drug, applying the Kaplan-Meier statistic. Progression was analysed according to the radiological criteria response evaluation criteria in solid tumours (RECIST V.1.1). The occurrence of adverse effects (AEs) leading to early dose reduction/suspension of the drug was analysed according to the common terminology criteria for adverse events (CTCAE).

Results 98 women (mean age 62.8 (34-86) years) were included in the study. $53.1 \%$ received $\mathrm{PB}(\mathrm{n}=52), 25.5 \%$ $(n=25)$ RB and 21.4\% $(n=21)$ AB. Mean follow-up time of the disease was 7.5 years. Mean duration of treatment was 9.5 months (0.1-34.3). 59.2\% $(\mathrm{n}=58)$ received previous adjuvant therapy, 20.4\% $(n=20)$ neoadjuvant and $74.5 \% \quad(n=73)$ hormonal.

Median PFS was AB vs PB (17.4 vs 11.9 months, $p=0.008)$; $A B$ vs $R B$ (17.4 months vs not reached, $p=0.065)$, $P B$ vs $R B$ $(\mathrm{p}=0.034) .34 .7 \% \quad(\mathrm{n}=34)$ suspended treatment due to progression and $15.3 \%(n=15)$ due to toxicity. $54.1 \%(n=53)$ of patients had doses reduced due to AEs during treatment: $\mathrm{PB}$ (45.3\%, $n=24), R B(24.5 \%, n=13)$ and $A B(30.2 \%, n=16)$. The most common AEs were: haematological (69.8\%, $\mathrm{n}=37$ ), gastrointestinal $(17 \%, \mathrm{n}=9)$, skin $(9.4 \%, \mathrm{n}=5)$, asthenic syndrome $(9.4 \%, \mathrm{n}=5)$, renal $(5.7 \%, \mathrm{n}=3)$, hepatic $(5.7, \mathrm{n}=3)$ and other $(3.8 \%, \mathrm{n}=2)$.

Conclusion and relevance PFS was more favourable for $\mathrm{AB}$ than for $\mathrm{PB}$, but not for RB. Likewise, RB treatment had a higher PFS than PB $(p \leq 0.05)$. More than $50 \%$ of patients had their dose reduced due to the appearance of AEs, with $\mathrm{PB}$ being the least tolerated. This highlights the need for close monitoring of these AEs, which impairs long term efficacy.

\section{REFERENCES AND/OR ACKNOWLEDGEMENTS}

Conflict of interest No conflict of interest 